\title{
A novel approach to Junior Doctor Induction: A near-peer based curriculum developed and delivered by outgoing Foundation year doctors
}

\author{
Kittiya Sukcharoen, Matthew Everson, Clare van Hamel
}

Severn Postgraduate Medical Education

\begin{abstract}
There is a 4-12\% increase in mortality in the month following the start of Foundation Year 1 doctors (FY1s) in the UK. In 2012 the National Health Service announced a compulsory shadowing period for FY1s, aiming to increase familiarity with the environment in which the FY1 would be commencing work. There is no national curriculum of the content for this shadowing period and evidence suggests variable content of induction programmes across the UK.
\end{abstract}

Our project aimed to provide a near-peer induction, based on needs previously identified by a national survey and outgoing FY1s' experiences. The day consisted of expert-led lectures, interactive practical sessions delivered by outgoing FY1s, and simulated tasks within the clinical environment where they were about to commence work. The day was evaluated by questionnaires distributed to participants before and after the induction to measure whether there was a change in the perceived confidence of the FY1s in different aspects of their role.

There was a $61 \%$ improvement in familiarity of equipment and knowing how to request investigations. Confidence levels increased by $45 \%$ and $28 \%$ in prescribing insulin and intravenous fluids, respectively. There was a $9 \%$ improvement in feeling adequately prepared to recognise the critically ill patient. Confidence was high in prescribing intravenous fluids ( $72 \%$ pre-induction and $100 \%$ post-induction) and simple analgesics (94\% pre-induction and $96 \%$ post-induction).

The induction day improved self-perceived confidence in all measured areas. The largest increase was in the area given most focus during the day - knowledge of the environment. Combining factual content with orientation of the environment increases confidence for new FY1s. Teaching by outgoing FY1s provides insight into what the job entails. We recommend this style of induction to maximise preparedness within a limited time frame.

\section{Problem}

There is a $4-12 \%$ reported increase in mortality in the month following the start of Foundation Year 1 doctors (FY1 - first year postgraduation) in the UK $(1,2)$. This has gained the media title of "Black Wednesday" as changeover occurs on the first Wednesday in August. Similar concerns have been reported in the USA and is known there as the "July phenomenon". The reported increase in mortality has been linked to the movement of most trainee doctors to unfamiliar environments around the UK on the same day. Lack of familiarity with the new working environment and unfamiliarity with local practices all contribute to doctors potentially feeling unprepared for their new jobs.

\section{Background}

The NHS announced a compulsory shadowing period for all FY1 doctors in 2013. Its aim was to improve knowledge and skills specific for the new working environment. Anecdotal evidence suggests a variety in duration and content of induction programmes across the UK. A combination of shadowing and teaching are usually included, but some hospitals have a 2-week induction programme while others have 4 days. There is currently no national curriculum although guidance was issued to trusts for the induction period. There is evidence that induction programmes can have an effect. University Hospitals Bristol NHS Foundation Trust showed a $45 \%$ reduction in self-reported critical incidents by new junior doctors in their first 4 months of working after introduction of mandatory structured induction training (3).

\section{Baseline measurement}

We ascertained the baseline measurement by gauging confidence levels at the start of the additional voluntary induction day, immediately before the 4-day shadowing period. There were 27 new FY1s starting at Great Western Hospital (GWH) in August 2013. Twenty-six of the 27 FY1s attended; pre- and post-induction feedback data from $25 \mathrm{FY} 1 \mathrm{~s}$ were collected.

The FY1s had high self-perceived confidence levels when it came to prescribing simple analgesics and intravenous fluids: more than $70 \%$ of FY1s felt confident. They also felt confident that they could recognise critically ill patients. Knowledge of the local environment (familiarity with equipment, knowing how to access investigation results) was very low. In addition, confidence was especially low concerning the prescribing of anticoagulants and insulin, with only $20 \%$ of FY1s feeling adequately prepared. 


\section{Design}

Our project aimed to provide near-peer induction, based on needs previously identified by a national survey of FY1s and local feedback from outgoing FY1s. The Preparedness to Practice Survey 2012, with 1829 FY1 responders, identified a number of areas where FY1s still felt unprepared after their induction (4). These included the local IT system, knowing the local equipment and environment, getting help out of hours, and prescribing.

The additional induction day was designed to address these areas of unpreparedness. The day consisted of:

- Expert-led lectures on diabetes, pharmacy, and acute oncology delivered by an endocrine consultant, a pharmacist, and an acute oncology specialist nurse.

- A sequential series of sessions, delivered by the outgoing FY1s, including fluid prescribing, sepsis, and use of the Foundation Programme e-portfolio.

- In-situ simulation where participants performed simulated tasks within the clinical environment where they were about to commence work: arterial blood gas sampling was simulated on the respiratory ward, death certification was completed in the bereavement office, and radiology requests were discussed with a radiology consultant in real time. The in situ simulation aimed to increase both knowledge but also environmental awareness and orientation.

- In the final session, students participated in classroom based simulation exercises in identifying and managing the unwell patient, commonly experienced while on call. This was run by an anaesthetic consultant.

\section{Strategy}

The aim of this project is to improve the confidence level of incoming FY1s, to orientate them around the new working environment, and to familiarise them with what to expect when they start work as an FY1. The ultimate aim is to improve patient safety.

The project started with anecdotal evidence drawn from surveys of current FY1s on their own experience of FY1 induction and what they would have liked to know before starting work. National surveys on FY1 induction were identified and reviewed. Research on different methods of teaching identified simulation as an effective tool in medical education and that in situ simulation is a new method of familiarising subjects to their new environments (5).

Based on the surveys and research on different teaching methods, we were able to draw up a provisional plan for the induction day, with key topics that must be covered. The outgoing FY1s participating on the day were then trained on simulation teaching formulating and writing scenarios, then running scenarios and debriefing students afterwards. The scenarios were then piloted with other FY1s to see if the scenarios were pitched at the right level and changes were made accordingly. The same methods were used in designing the scenarios for in situ simulation.
Respective departments were approached and agreed what they thought might be useful scenarios; some recommendations were later amended or rejected after being piloted on the current FY1s. Consideration was given to piloting scenarios with final year students but this was not possible due to local students being on their elective during the scenario testing period. The speakers for the expert led lectures were consultants or specialist nurses who have done talks on these topics before to a similar audience.

After the voluntary induction day was delivered, we collated the results from the feedback questionnaires and the results were presented to an audience including the Trust chief executive, medical director and education leads at GWH. The presentation highlighted the importance of FY1 induction, which is specific to the environment in which FY1s will be working. There was agreement that using a near-peer approach with outgoing FY1s had delivered positive feedback. The Trust has agreed to incorporate the induction programme into the compulsory Trust induction for new FY1s. The induction programme for 2014 will be evaluated to try to ensure an improvement in the confidence and preparedness of the incoming FY1s.

\section{Results}

The environmental induction day was evaluated by questionnaires distributed to the 26 participants before and after the induction; $96 \%$ of attendees completed the evaluation. The self-perceived change in confidence was measured.

There was a $61 \%$ improvement in familiarity of equipment; a $61 \%$ improvement in knowing how to request investigations; a $46 \%$ improvement in familiarity with the e-portfolio; and a $45 \%$ improvement in knowledge of the working environment (see figure attached). Confidence levels were high in knowing what to expect of starting FY1s which improved from $64 \%$ to $88 \%$ after the day. There was a $9 \%$ improvement in feeling adequately prepared to recognise the critically ill patient, from a high baseline of $72 \%$ feeling confident.

Regarding prescribing, confidence levels increased the most, by $45 \%$, in prescribing insulin. Confidence was high in prescribing intravenous fluids (72\% pre-induction and $100 \%$ post-induction) and simple analgesics (94\% pre-induction and $96 \%$ post-induction).

Compared to other FY1s across the UK in the National Preparedness to Practice Survey 2013, FY1s at the GWH all had an opportunity to shadow the outgoing $\mathrm{FY} 1 \mathrm{~s}$ ( $85 \%$ vs $100 \%$ ). More FY1s at GWH had taken a tour of the working environment, had teaching on critically ill patients and FY1 e-portfolio compared to national figures. The survey also demonstrated an overall improvement in confidence compared to the national average and an improvement on the 2012 national survey results for the GWH.

See supplementary file: ds3721.pptx - "Graph"

\section{Lessons and limitations}




\section{BMJ Quality Improvement Reports}

We collected subjective data, notably self-perceived confidence, and it should be noted this does not necessarily reflect actual knowledge and skills.

Interestingly, the new FY1s were confident in their ability to recognise the critically ill patient. However, research has highlighted concerns regarding the paucity of understanding in final year medical students of the management of critical illness (6). The high confidence in our $\mathrm{F} 1 \mathrm{~s}$ could be explained by their recent final medical school examinations. The new doctors may have confused their knowledge of the critically ill with the experience of being able to recognise and manage such a patient.

We have concerns over the sustainability of our project given that it was led by FY1 doctors. We are pleased to say it is being repeated this year. This was a 1-day induction programme provided in addition to the compulsory existing induction at the Trust. Integration within the mandatory induction programme would provide greater certainty of sustainability of the project. The day was $10 \mathrm{~h}$ long and FY1s commented that this was too long to spend learning intensively. Ideally, this programme should be implemented over 2 days. However, this would involve more organisation and taking time out of the mandatory Trust induction programme.

The day was cost neutral, with lunch supplied by a sponsor. The burden of tutor availability and having to take time away from clinical work was not found to be an issue.

\section{Conclusion}

The induction day improved self-perceived confidence in all measured areas. The largest increase in confidence was in the area given greatest focus during the day, namely knowledge of the environment. Combining factual content with orientation of the environment increases confidence for new FY1s. Teaching by outgoing FY1s provides insight into what the job entails. We recommend this style of induction to maximise preparedness with limited time.

\section{References}

1. Young J, Ranji S, Wachter R, et al "July effect": impact of the academic year-end changeover on patient outcomes: a systematic review. Ann Intern Med 2011;155:309-15.

2. Jen MH, Bottle A, Majeed A, Bell D, Aylin P. Early inhospital mortality following trainee doctors' first day at work. PLoS ONE 2009;4(9):e7103.

3. Aspinall R, Blencowe N. Improving patient safety. Transition between finals and the first night shift. University Hospitals Bristol NHS Foundation Trust. Presented at the International Forum on Patient Safety (Berlin, March 2009) and the Association for Medical Education in Europe (Malaga, September 2009).

4. van Hamel C. Preparedness to Practice National Survey. National Association of Clinical Tutors Conference. (London).

5. Patterson, M, Blike, G, Nadkarni, V. In situ simulation: challenges and results. Advances In Patient Safety 2008;2(3):1-18.

6. Kelly, D. The knowledge of medical students and newly qualified doctors concerning the specialty of intensive care medicine. Journal of Intensive Care Society 2011;12:98-106

\section{Declaration of interests}

Clare van Hamel is Clinical Advisor to the UKFPO.

\section{Acknowledgements}

Mia Kahvo, Victoria Taylor, Alex Brooks-Moizer 